\title{
Dividends Sharing Convertible Bonds Pricing and Numerical Evaluation
}

\author{
$\mathrm{Xu} \mathrm{Guo}^{1}$ and Haiyang Wang ${ }^{2}$ \\ ${ }^{1}$ Department of Mathematics, Hong Kong Baptist University, Kowloon Tong, Hong Kong \\ ${ }^{2}$ School of Mathematics, Shandong University, Jinan 250100, China \\ Correspondence should be addressed to Haiyang Wang; health.sea@163.com
}

Received 23 January 2013; Accepted 23 April 2013

Academic Editor: Guangchen Wang

Copyright (c) 2013 X. Guo and H. Wang. This is an open access article distributed under the Creative Commons Attribution License, which permits unrestricted use, distribution, and reproduction in any medium, provided the original work is properly cited.

\begin{abstract}
The convertible bond is becoming one of the most important financial instruments for the company to raise capital fund since it was first issued by American New York Erie Company in 1843. In this paper, it is the first time to study the pricing problem for convertible bond whose underlying stocks pay dividends via the reflected backward stochastic differential equations. Associating the solutions of reflected BSDEs with the obstacle problems for nonlinear parabolic PDEs, we establish the pricing formulas for convertible bonds with continuous and discrete dividends by means of the viscosity solutions for some PDEs. Besides, we also derive the price of convertible bonds with higher borrowing rate which is realistic in the financial market. Then the numerical evaluations are provided by the radial basis functions method. Moreover, we discuss the influence of dividends paying as well as higher borrowing rate on the convertible bond price at last.
\end{abstract}

\section{Introduction}

A convertible bond is a kind of corporate debt securities that gives the holder the right to forgo future coupon and principal payments and convert it to a specified number of shares of common stock of the issuing firm. In essence, a convertible bond is a hybrid security consisting of a straight bond and a call on the underlying equity, but various characteristics of realistic convertible bonds make it impossible to decouple the stock option from the bond part. Therefore, how to price the convertible bond fairly is more difficult than many other derivatives.

Theoretical pricing models for convertible bonds first appeared in the 1960s. Their general valuation procedure was to set the price of the convertible bond equal to the maximum of its value as an ordinary bond or its value in common stock (after conversion) at some point in the future and then discount this value to the present. This method or a slight modification thereof was employed by Poensgen $[1,2]$, Baumol et al. [3], Weil et al. [4], and no doubt others. Since the fundamental paper worked by Black and Scholes [5] for pricing the financial derivatives was published in 1973, the celebrated Black-Scholes formula is adopted to value the convertible bond as a contingent claim on the firm as a whole. There is also a rich literature along this line, for example, Ingersoll [6] and Brennan and Schwartz $[7,8]$, in all of which the authors took the firm value as the variable that determine the price of the convertible bond, while in McConnell and Schwartz [9], Ho and Pfeffer [10], and Tsiveriotis and Fernandes [11], the convertible bond is viewed and valued as a derivative of the underlying equity, which is commonly the stock of the issuing firm.

However, the backward stochastic differential equations (BSDEs) are a powerful alternative to price the contingent claims. Nonlinear BSDEs were introduced by Pardoux and Peng [12] and Duffie and Epstein [13] independently. El Karoui et al. [14] studied the property of BSDEs and their applications to optimal control and financial mathematics, such as European option pricing problem in the constraint case. Since the price of convertible bond is constrained to be greater than the payoff of the bond, it corresponds to the solution of a new type of backward equations called reflected BSDEs. El Karoui et al. [15] investigated the RBSDEs in detail and gave some important properties, among which is the connection between the solutions of RBSDEs and the related obstacle problems for parabolic PDEs. Our work is much inspired by them and attempts to price the convertible bonds 
with continuous and discrete dividends as well as with higher borrowing rate.

Despite the difficulty of obtaining the analytic solutions of nonlinear parabolic PDEs, the radial basis functions (RBFs) play an important role in solving different types of PDEs numerically with many advantages such as mesh-free property, accuracy, stability, and efficiency. As one of notable RBFs, Hardy's Multiquadric (MQ) was firstly developed by Hardy [16] to approximate two-dimensional geographical surfaces. In Franke's [17] review paper, the MQ was rated one of the best methods among 29 scattered data interpolation schemes. Hon and Mao [18] applied this MQ as a spatial approximation to devise a new computation scheme for the numerical solution of the options value and its derivatives in the Black-Scholes equation. This transforms the BlackScholes equation to a system of first-order equations in time, and the American options can then be approximated by using a high-order backward time integration scheme. In our paper, we adopt this kind of methods to evaluate the PDEs for pricing the convertible bonds, which makes our theoretical results more practical in the financial market.

The rest of this paper is organized as follows. We introduce some key characteristics of a convertible bond and recall the definition of reflected BSDEs and some important properties of it in Section 2. In Section 3, we establish the pricing formula for convertible bonds whose underlying stocks pay continuous and discrete dividends, respectively, by means of nonlinear parabolic PDEs. Furthermore, we investigate the situation with higher borrowing rate in Section 4. Then according to the computation scheme of RBF methods, some examples with all constant coefficients are presented in Section 5, and we can also see the influence of dividends paying as well as higher borrowing rate on the convertible bond price through the numerical results. The last section is devoted to conclude the novelty and distinctive feature of the paper and discuss the future research work in this field.

\section{Preliminaries and Notations}

In this section, at first, let us get familiar with some unique characteristics of the convertible bond and then recall some properties of reflected BSDEs and the associated obstacle problems for nonlinear parabolic PDEs.

2.1. A Convertible Bond Indenture Agreement. In finance, a convertible bond is a type of bond that the holder can convert into shares of common stock in the issuing company or cash of equal value, at an agreed-upon price. It is a hybrid security with both debt- and equity-like features. Although it typically has a coupon rate lower than that of similar, nonconvertible debt, the instrument carries additional value through the option to convert the bond to stock and thereby participates in further growth in the company's equity value. The investor receives the potential upside of conversion into equity while protecting downside with cash flow from the coupon payments and the return of principal upon maturity.

From the issuer's perspective, the key benefit of raising money by selling convertible bonds is a reduced cash interest payment. The advantage for companies of issuing convertible bonds is that, if the bonds are converted to stocks, companies' debt vanishes. However, in exchange for the benefit of reduced interest payments, the value of shareholder's equity is reduced due to the stock dilution expected when bondholders convert their bonds into new shares.

Generally speaking, there are several articles that determine the feature of a convertible bond in the indenture agreement. They are as follows.

(i) Maturity Date $T$ : it is the date from now to which a holder can convert the bond to a specified number of common stocks at any time during this period, that is, the expiry date of a convertible bond.

(ii) Conversion Ratio $C$ : it states the number of shares of common stock which can be obtained upon the surrender of one share of convertible bond.

(iii) Face Price F: it is the aggregate balloon payment that an investor can get at the maturity date $T$ if he never exercises the convertible bond.

(iv) Put Term: it is an agreement that on each put date the investor can choose between holding the convertible bond and putting it to the issuer for the specified put value.

(v) Call Term: it is an agreement that when the issuer calls the convertible bond, the investor must elect to receive either the cash call price or the conversion value of the convertible bond.

2.2. Reflected Backward Stochastic Differential Equations. Let $(\Omega, \mathscr{F}, P)$ be a completed probability space endowed with a filtration $\left\{\mathscr{F}_{t} ; 0 \leq t \leq T\right\}$. Also $\left\{W_{t}, 0 \leq t \leq T\right\}$ is a $d$-dimensional standard Brownian motion defined on this space. We also assume that $\left\{\mathscr{F}_{t}\right\}_{t \geq 0}$ is generated by the Brownian motion $\left\{W_{t}, t \geq 0\right\}$ and satisfies the usual conditions. If $x$ belongs to $\mathbb{R}^{n},|x|$ denotes its Euclidean norm.

There are some notations used throughout the paper:

$$
\begin{aligned}
& \mathscr{L}^{2}\left(\mathscr{F}_{T}, \mathbb{R}^{m}\right) \\
& =\left\{\xi \text { is a } \mathbb{R}^{m} \text {-valued } \mathscr{F}_{T}\right. \text {-measurable random variable } \\
& \left.\quad \text { s.t. } \mathbb{E}\left(|\xi|^{2}\right)<+\infty\right\}, \\
& \mathscr{L}_{\mathscr{F}}^{2}\left(0, T ; \mathbb{R}^{m}\right) \\
& =\left\{\left\{\varphi_{t}, 0 \leq t \leq T\right\} \text { is a } \mathbb{R}^{m}\right. \text {-valued adapted process } \\
& \left.\quad \text { s.t. } \mathbb{E}\left(\int_{0}^{T}\left|\varphi_{t}\right|^{2} d t\right)<+\infty\right\}, \\
& \mathcal{S}_{\mathscr{F}}^{2}\left(0, T ; \mathbb{R}^{m}\right) \\
& =\left\{\left\{\psi_{t}, 0 \leq t \leq T\right\} \text { is a } \mathbb{R}^{m}\right. \text {-valued adapted process } \\
& \left.\quad \text { s.t. } \mathbb{E}\left(\sup _{0 \leq t \leq T}\left|\psi_{t}\right|^{2}\right)<+\infty\right\} .
\end{aligned}
$$


Let us introduce the 1-dimensional reflected BSDEs. We are given three objects: the first is a terminal value $\xi$ s.t.

(i) $\xi \in \mathscr{L}^{2}\left(\mathscr{F}_{T}, \mathbb{R}\right)$.

The second is a "coefficient" $f$, which is a map

$$
f: \Omega \times[0, T] \times \mathbb{R} \times \mathbb{R}^{d} \longrightarrow \mathbb{R},
$$

such that

(ii) for all $(y, z) \in \mathbb{R} \times \mathbb{R}^{d}, f(\cdot, y, z) \in \mathscr{L}_{\mathscr{F}}^{2}(0, T ; \mathbb{R})$,

(iii) for some $K>0$ and all $y, y^{\prime} \in \mathbb{R}, z, z^{\prime} \in \mathbb{R}^{d}$, a.s.,

$$
\left|f(t, y, z)-f\left(t, y^{\prime}, z^{\prime}\right)\right| \leq K\left(\left|y-y^{\prime}\right|+\left|z-z^{\prime}\right|\right) .
$$

The third is an "obstacle" $\left\{S_{t} ; 0 \leq t \leq T\right\}$, which is a continuous progressively measurable real-valued process satisfying

(iv) $\mathbb{E}\left(\sup _{0 \leq t \leq T}\left(S_{t}^{+}\right)^{2}\right)<\infty$.

We will always assume that $S_{T} \leq \xi$ a.s.

Then we formulate the form of a reflected BSDE.

Definition 1. The solution of the reflected BSDE is a triple $\left\{\left(Y_{t}, Z_{t}, K_{t}\right), 0 \leq t \leq T\right\}$ of $\mathscr{F}_{t}$-progressively measurable processes taking values in $\mathbb{R}, \mathbb{R}^{d}$, and $\mathbb{R}_{+}$, respectively, and satisfying

(1) $Y \in \mathcal{S}_{\mathscr{F}}^{2}(0, T ; \mathbb{R}), Z \in \mathscr{L}_{\mathscr{F}}^{2}\left(0, T ; \mathbb{R}^{d}\right)$, and $K_{T} \in$ $\mathscr{L}^{2}\left(\mathscr{F}_{T}, \mathbb{R}\right)$,

(2) $Y_{t}=\xi+\int_{t}^{T} f\left(s, Y_{s}, Z_{s}\right) d s+K_{T}-K_{t}-\int_{t}^{T} Z_{s} d W_{s}$,

(3) $Y_{t} \geq S_{t}, 0 \leq t \leq T$,

(4) $\left\{K_{t}\right\}$ is continuous and increasing, $K_{0}=0$, and $\int_{0}^{T}\left(Y_{t}-\right.$ $\left.S_{t}\right) d K_{t}=0$.

The existence and uniqueness of solutions of reflected BSDEs have been proved by El Karoui et al. [15].

Theorem 2. Under the previous assumptions, in particular (i), (ii), (iii), and (iv), the reflected BSDE with (1), (2), (3), and (4) has a unique solution $(Y, Z, K)$.

Moreover, some properties of the reflected BSDEs are also summarized and presented there. An important fact is that the square-integrable solution $\left\{Y_{t}, 0 \leq t \leq T\right\}$ of reflected BSDE corresponds to the value of an optimal stopping time problem.

Proposition 3. Let $\left\{\left(Y_{t}, Z_{t}, K_{t}\right), 0 \leq t \leq T\right\}$ be the solution of reflected BSDE defined by Definition 1 . Then for each $t \in[0, T]$,

$$
Y_{t}=\operatorname{ess} \sup _{v \in \mathscr{T}_{t}} \mathbb{E}\left[\int_{t}^{v} f\left(s, Y_{s}, Z_{s}\right) d s+S_{v} \mathbf{1}_{\{v<T\}}+\xi \mathbf{1}_{\{v=T\}} \mid \mathscr{F}_{t}\right],
$$

where $\mathscr{T}$ is the set of all stopping times dominated by $T$, and

$$
\mathscr{T}_{t}=\{v \in \mathscr{T} ; t \leq v \leq T\}
$$

2.3. Obstacle Problems for Nonlinear Parabolic PDEs. In this subsection, we will show that the reflected BSDEs allow us to give a probabilistic representation for solutions of some obstacle problems for PDEs. For that purpose, we will put the RBSDEs in a Markovian framework.

Let $b:[0, T] \times \mathbb{R}^{d} \rightarrow \mathbb{R}^{d}$ and $\sigma:[0, T] \times \mathbb{R}^{d} \rightarrow \mathbb{R}^{d \times d}$ be continuous mappings, which are Lipschitz with respect to their second variable, uniformly with respect to $t \in[0, T]$. For each $(t, x) \in[0, T] \times \mathbb{R}^{d}$, let $\left\{X_{s}^{t, x}, t \leq s \leq T\right\}$ be the unique $\mathbb{R}^{d}$-valued solution of the SDE:

$$
X_{s}^{t, x}=x+\int_{t}^{s} b\left(r, X_{r}^{t, x}\right) d r+\int_{t}^{s} \sigma\left(r, X_{r}^{t, x}\right) d W_{r}
$$

We suppose now that the data $(\xi, f, S)$ of the RBSDE take the following form:

$$
\begin{gathered}
\xi=g\left(X_{T}^{t, x}\right), \\
f(s, y, z)=f\left(s, X_{s}^{t, x}, y, z\right), \\
S_{s}=h\left(s, X_{s}^{t, x}\right),
\end{gathered}
$$

where $g, f$, and $h$ are as follows. First, $g \in C\left(\mathbb{R}^{d}\right)$ has at most polynomial growth at infinity. Second,

$$
f:[0, T] \times \mathbb{R}^{d} \times \mathbb{R} \times \mathbb{R}^{d} \longrightarrow \mathbb{R}
$$

is jointly continuous and for some $K>0, p \in \mathbb{N}$, satisfies

$$
\begin{gathered}
|f(t, x, 0,0)| \leq K\left(1+|x|^{p}\right), \\
\left|f(t, x, y, z)-f\left(t, x, y^{\prime}, z^{\prime}\right)\right| \leq K\left(\left|y-y^{\prime}\right|+\left|z-z^{\prime}\right|\right),
\end{gathered}
$$

for $t \in[0, T], x, z, z^{\prime} \in \mathbb{R}^{d}, y, y^{\prime} \in \mathbb{R}$. Finally,

$$
h:[0, T] \times \mathbb{R}^{d} \longrightarrow \mathbb{R}
$$

is jointly continuous in $t$ and $x$ and satisfies

$$
h(t, x) \leq K\left(1+|x|^{p}\right), \quad t \in[0, T], x \in \mathbb{R}^{d} .
$$

We assume moreover that $h(T, x) \leq g(x), x \in \mathbb{R}^{d}$.

For each $t>0$, we denote by $\left\{\mathscr{F}_{s}^{t}, t \leq s \leq T\right\}$ the natural filtration of Brownian motion $\left\{B_{s}-B_{t}, t \leq s \leq T\right\}$, argumented by the $P$-null sets of $\mathscr{F}$.

It follows from Theorem 2 that for each $(t, x)$ there exists a unique triple $\left(Y^{t, x}, Z^{t, x}, K^{t, x}\right)$ of $\mathscr{F}_{s}^{t}$-progressively measurable processes, which solves the following RBSDE:

(1) $\mathbb{E} \int_{t}^{T}\left(\left|Y_{s}^{t, x}\right|^{2}+\left|Z_{s}^{t, x}\right|^{2}\right) d s<\infty$,

(2) $Y_{s}^{t, x}=g\left(X_{T}^{t, x}\right)+\int_{s}^{T} f\left(r, X_{r}^{t, x}, Y_{r}^{t, x}, Y_{r}^{t, x}\right) d r+K_{T}^{t, x}-$ $K_{s}^{t, x}-\int_{s}^{T} Z_{r}^{t, x} d W_{r}, t \leq s \leq T$,

(3) $Y_{s}^{t, x} \geq h\left(s, X_{s}^{t, x}\right), 0 \leq t \leq T$,

(4) $\left\{K_{s}^{t, x}\right\}$ is continuous and increasing, and $\int_{t}^{T}\left(Y_{s}^{t, x}-\right.$ $\left.h\left(s, X_{s}^{t, x}\right)\right) d K_{s}^{t, x}=0$. 
We now consider the related obstacle problem for a parabolic PDE. Roughly speaking, a solution of the obstacle problem is a function $u:[0, T] \times \mathbb{R}^{d} \rightarrow \mathbb{R}$ which satisfie

$$
\begin{gathered}
\min \left(u(t, x)-h(t, x),-\frac{\partial u}{\partial t}(t, x)-L_{t} u(t, x)\right. \\
-f(t, x, u(t, x),(\nabla u \sigma)(t, x)))=0, \\
(t, x) \in(0, T) \times \mathbb{R}^{d}, \\
u(T, x)=g(x), \quad x \in \mathbb{R}^{d},
\end{gathered}
$$

where

$$
L_{t}=\frac{1}{2} \sum_{i, j=1}^{d}\left(\sigma \sigma^{*}(t, x)\right)_{i, j} \frac{\partial^{2}}{\partial x_{i} \partial x_{j}}+\sum_{i=1}^{d} b_{i}(t, x) \frac{\partial}{\partial x_{i}} .
$$

We now define

$$
u(t, x) \triangleq Y_{t}^{t, x}, \quad(t, x) \in[0, T] \times \mathbb{R}^{d},
$$

which is a deterministic quantity; then we have the following result (see, e.g., El Karoui et al. [15]).

Theorem 4. Defined by $(14), u(t, x)$ is the unique viscosity solution of the obstacle problem (12).

\section{Pricing Model with Dividends Paying}

Suppose that there are two kinds of assets in a financial market: one is the bank account which obeys

$$
d B_{s}=r_{s} B_{s} d s
$$

the other is the stock of the company with price process

$$
d P_{s}=\mu_{s} P_{s} d s+\sigma_{s} P_{s} d W_{s},
$$

where $r_{t}$ is the riskless interest rate and $\mu_{t}$ and $\sigma_{t}$ are the expected interest rate and volatility rate of the stock, respectively.

Throughout the paper, we need the following assumptions for the market.

Assumption 5. Suppose that the financial market is like this.

(1) The capital markets are perfect with no transactions costs, no taxes, and equal access to information for all investor.

(2) The convertible bonds are not allowed to be called or putted, and the issuer will not default.

(3) The riskless interest rate $r_{t}$, the expected interest rate $\mu_{t}$, and the volatility rate $\sigma_{t}$ of the company's stock are all deterministic and bounded; $\sigma_{t}$ is invertible, and the inverse $\sigma_{t}^{-1}$ is also bounded.

(4) The convertible bond can be converted before any time of the maturity date $T$, and the conversion ratio $C$ is a constant.
For any convertible bond in this financial market, its underlying stocks may pay dividends continuously or discretely. We will establish the pricing formulas for both cases in this section.

3.1. The Continuous Dividends Case. Generally speaking, the dividends can be influenced by many external factors, such as the underlying stock price, enterprise profits, and developing strategies. Nevertheless, under some circumstances, for example, when the stock price is relatively low or the stock is short after its going-to-market, the stock price is the elementary factor affecting the dividends paying. We study such a case in this subsection and need the following assumption.

Assumption 6. The dividends of the underlying stocks is paid continuously at rate $d\left(t, P_{t}\right)$, where $d(t, \cdot)$ is deterministic and bounded.

Thus, a convertible bond is similar to an American call option with the terminal value $\xi=\max \left(C P_{T}, F\right)$ and the "obstacle" process $\left\{C P_{t}, 0 \leq t \leq T\right\}$. So we adopt the methods for pricing American options employed by El Karoui et al. [19]. At each time $t$, pricing a convertible bond consists of the selection of a stopping time $v \in \mathscr{T}_{t}$ and a payoff $C P_{v}$ on exercise if $v<T$ and $\xi$ if $v=T$. Denote

$$
\widetilde{P}_{s}=C P_{s} \mathbf{1}_{\{s \leq T\}}+\xi \mathbf{1}_{\{s=T\}},
$$

and fix $t \in[0, T]$. Suppose for a moment that the choice of $v \in \mathscr{T}_{t}$ has been made. Then there exists a unique hedging portfolio strategy $\left(X_{s}\left(v, \widetilde{P}_{v}\right), \pi_{s}\left(v, \widetilde{P}_{v}\right)\right) \in \mathcal{S}_{\mathscr{F}}^{2} \times \mathscr{L}_{\mathscr{F}}^{2}$, denoted also by $\left(X_{s}^{v}, \pi_{s}^{v}\right)$, which replicates $\widetilde{P}_{v}$, that is, the solution of the classical BSDE associated with the terminal time $v$, terminal value $\widetilde{P}_{v}$ :

$$
\begin{gathered}
d X_{s}^{v}=\left[r_{s} X_{s}^{v}+\left(\mu_{s}-r_{s}+d\left(s, P_{s}\right)\right) \pi_{s}^{v}\right] d s+\sigma_{s} \pi_{s}^{v} d W_{s}, \\
X_{v}^{v}=\widetilde{P}_{v} .
\end{gathered}
$$

Set

$$
Z_{s}^{v}=\sigma_{s} \pi_{s}^{v}, \quad \theta_{s}=\sigma_{s}^{-1}\left(\mu_{s}-r_{s}+d\left(s, P_{s}\right)\right),
$$

and thus (18) can be rewritten as

$$
\begin{gathered}
-d X_{s}^{v}=-\left(r_{s} X_{s}^{v}+\theta_{s} Z_{s}^{v}\right) d s-Z_{s}^{v} d W_{s}, \\
X_{v}^{v}=\widetilde{P}_{v} .
\end{gathered}
$$

Then, the price of the convertible bond at time $t$ is given by the right-continuous adapted process $\left\{X_{t}^{c}, 0 \leq t \leq T\right\}$ satisfying

$$
X_{t}^{c}=\operatorname{ess} \sup _{v \in \mathscr{T}_{t}} X_{t}\left(v, \widetilde{P}_{v}\right)
$$

By Proposition 3, it follows that the price process $\left\{X_{t}^{c}, 0 \leq t \leq\right.$ $T\}$ corresponds to the solution of a reflected BSDE. 
Theorem 7. Let Assumptions 5 and 6 hold. Then there exist $\left(Z_{t}^{c}\right) \in \mathscr{L}_{\mathscr{F}}^{2}$ and $\left(K_{t}^{c}\right)$ an increasing adapted continuous process with $K_{0}^{c}=0$ such that

$$
\begin{array}{r}
X_{t}^{c}=\xi+\int_{t}^{T}-\left(r_{s} X_{s}^{c}+\theta_{s} Z_{s}^{c}\right) d s+K_{T}^{c}-K_{t}^{c}-\int_{t}^{T} Z_{s}^{c} d W_{s} \\
0 \leq t \leq T,
\end{array}
$$

where $X_{t}^{c} \geq C P_{t}, 0 \leq t \leq T$, and $\int_{0}^{T}\left(X_{t}^{c}-C P_{t}\right) d K_{t}^{c}=0$.

Furthermore, the stopping time $D_{t}=\inf \left\{t \leq s \leq T ; X_{s}=\right.$ $\left.C P_{s}\right\}$ is the execution time of the convertible bond; that is

$$
X_{t}^{c}=\operatorname{ess} \sup _{v \in \mathscr{T}_{t}} X_{t}\left(v, \widetilde{P}_{v}\right)=X_{t}\left(D_{t}, \widetilde{P}_{D_{t}}\right) .
$$

The process $\left\{K_{t}^{c}, 0 \leq t \leq T\right\}$ may be interpreted as a cumulative consumption process. Such a triple $\left\{\left(Y_{t}^{c}, Z_{t}^{c}, K_{t}^{c}\right), 0 \leq\right.$ $t \leq T\}$ satisfying (22) with $X_{t}^{c} \geq C P_{t}, 0 \leq t \leq T$, (but not necessarily $\left.\int_{0}^{T}\left(X_{t}^{c}-C P_{t}\right) d K_{t}^{c}=0\right)$ is called a superhedging strategy for the convertible bond $\left(C P_{t}, \xi\right)$. Consequently, the price $X_{t}^{c}$ is equal to the so-called "upper price" defined as the smallest of the superhedging strategies for $\left(C P_{t}, \xi\right)$.

Noticing the relationship between the solutions of reflected BSDEs and the obstacle problems for nonlinear parabolic PDEs, according to Theorem 4, the convertible bond price process $\left\{X_{t}^{c}, 0 \leq t \leq T\right\}$ is also the viscosity solution of a PDE.

Theorem 8. Let Assumptions 5 and 6 hold. Then the following PDE

$$
\begin{gathered}
\min \left(u(t, x)-C x, \frac{\partial u}{\partial t}(t, x)+\frac{1}{2} \sigma_{t}^{2} x^{2} \frac{\partial^{2} u}{\partial x^{2}}(t, x)\right. \\
\left.+\left(r_{t}-d(t, x)\right) x \frac{\partial u}{\partial x}(t, x)-r_{t} u(t, x)\right)=0, \\
(t, x) \in(0, T) \times \mathbb{R}, \\
u(T, x)=\max (C x, F), \quad x \in \mathbb{R},
\end{gathered}
$$

admits a unique viscosity solution $u(t, x)$. The convertible bond price process $\left\{X_{t}, 0 \leq t \leq T\right\}$ can be given as

$$
X_{t}=u\left(t, P_{t}\right), \quad \forall t \in[0, T],
$$

where $P_{t}$ is the price of underlying stock at time $t$.

3.2. The Discrete Dividends Case. In a real financial market, the stock dividends are usually paid discretely at some future dates. Simultaneously, the stock price will fall down. More precisely, we suppose the following.

Assumption 9. The dividends is paid in the proportion $\delta$ of the stock price at some prefixed future dates $T_{1}, T_{2}, \ldots, T_{n}$ before the maturity date $T$.

Recalling the practical effects of dividends paying to the stock price, we suppose $P_{s}$ admits

$$
d P_{s}=\mu_{s} P_{s} d s+\sigma_{s} P_{s} d W_{s}+d L_{s},
$$

where $L_{s}$ is a decreasing deterministic left continuous step function with jumps $\delta P_{T_{i}}$ at $T_{i}, i=1,2, \ldots, n$, initialized by 0 .

For simplicity and convenience, we first confine ourselves to the case of a single dividends paying before the maturity date temporarily. Correspondingly, $L_{s}$ is a decreasing deterministic left continuous step function with jump $\delta P_{T_{1}}$ at $T_{1}<$ $T$, starting at 0 .

Thus, the price process (26) of the underlying stock consists of two stages:

$$
\begin{gathered}
d P_{s}=\mu_{s} P_{s} d s+\sigma_{s} P_{s} d W_{s}, \quad 0 \leq s \leq T_{1}, \\
P_{0}=p_{0}, \\
d P_{s}=\mu_{s} P_{s} d s+\sigma_{s} P_{s} d W_{s}, \quad T_{1}<s \leq T, \\
P_{T_{1+}}=(1-\delta) P_{T_{1}} .
\end{gathered}
$$

Firstly, we calculate the value of convertible bond $\left(X_{t}^{d}\right)$ on the time interval $\left(T_{1}, T\right]$. Similar to the analysis in Section 3.1, the price process $\left\{X_{t}^{d}, T_{1}<t \leq T\right\}$ satisfies

$$
\begin{aligned}
X_{t}^{d}= & +\int_{t}^{T}-\left[\left(r_{s} X_{s}^{d}+\sigma_{s}^{-1}\left(\mu_{s}-r_{s}\right) Z_{s}^{d}\right)\right] d s \\
& +K_{T}^{d}-K_{t}^{d}-\int_{t}^{T} Z_{s}^{d} d W_{s}, \quad T_{1}<t \leq T,
\end{aligned}
$$

where $X_{t}^{d} \geq C P_{t}, T_{1}<t \leq T$, and $\int_{T_{1}}^{T}\left(X_{t}^{d}-C P_{t}\right) d K_{t}^{d}=0$.

Associating (29) with (28), by Theorem 4, we get the evaluation of PDE

$$
\begin{gathered}
\min \left(u^{1}(t, x)-C x, \frac{\partial u^{1}}{\partial t}(t, x)+\frac{1}{2} \sigma_{t}^{2} x^{2} \frac{\partial^{2} u^{1}}{\partial x^{2}}(t, x)\right. \\
\left.+r_{t} x \frac{\partial u^{1}}{\partial x}(t, x)-r_{t} u^{1}(t, x)\right)=0 \\
(t, x) \in\left(T_{1}, T\right) \times \mathbb{R} \\
u^{1}(T, x)=\max (C x, F), \quad x \in \mathbb{R}
\end{gathered}
$$

Thus, the convertible bond price $X_{t}^{d}=u^{1}\left(t, P_{t}\right)$ during the time $\left(T_{1}, T\right]$.

Secondly, we consider the situation on $\left[0, T_{1}\right]$. By (28), we know $X_{T_{1+}}^{d}=u^{1}\left(T_{1},(1-\delta) P_{T_{1}}\right)$. At the dividends paying date $T_{1}$, any reasonable investor will compare the value of converted stocks and the convertible bond and then decide whether to execute it or not. Again similarly to before, denote

$$
\xi_{1}=\max \left(C P_{T_{1}}, u^{1}\left(T_{1},(1-\delta) P_{T_{1}}\right)\right)
$$

Then, the convertible bond price process $\left\{X_{t}^{d}, 0 \leq t \leq T_{1}\right\}$ is given by

$$
\begin{aligned}
X_{t}^{d}= & \xi_{1}+\int_{t}^{T_{1}}-\left[\left(r_{s} X_{s}^{d}+\sigma_{s}^{-1}\left(\mu_{s}-r_{s}\right) Z_{s}^{d}\right)\right] d s \\
& +K_{T_{1}}^{d}-K_{t}^{d}-\int_{t}^{T_{1}} Z_{s}^{d} d W_{s}, \quad 0 \leq t \leq T_{1},
\end{aligned}
$$

where $X_{t}^{d} \geq C P_{t}, 0 \leq t \leq T_{1}$, and $\int_{0}^{T_{1}}\left(X_{t}^{d}-C P_{t}\right) d K_{t}^{d}=0$. 
Also by Theorem 4, the evaluation of PDE is easily derived that

$$
\begin{gathered}
\min \left(u^{0}(t, x)-C x, \frac{\partial u^{0}}{\partial t}(t, x)+\frac{1}{2} \sigma_{t}^{2} x^{2} \frac{\partial^{2} u^{0}}{\partial x^{2}}(t, x)\right. \\
\left.+r_{t} x \frac{\partial u^{0}}{\partial x}(t, x)-r_{t} u^{0}(t, x)\right)=0, \\
(t, x) \in\left(0, T_{1}\right) \times \mathbb{R}, \\
u^{0}\left(T_{1}, x\right)=\max \left(C x, u^{1}\left(T_{1},(1-\delta) x\right)\right), \quad x \in \mathbb{R},
\end{gathered}
$$

and the fair price of convertible bond $X_{t}^{d}=u^{0}\left(t, P_{t}\right)$ during the time $\left[0, T_{1}\right]$, with $P_{t}$ to be the stock price at time $t$.

Therefore, the convertible bond price can be calculated via two PDEs (30) and (33) which may be solved successively.

Analogously, for the general case where dividends paying are in the proportion $\delta$ of the stock price at dates $T_{1}, T_{2}, \ldots, T_{n}$ before the maturity date $T$, the convertible bond price is obtained via the following series of PDEs:

$$
\begin{gathered}
\min \left(u^{n}(t, x)-C x, \frac{\partial u^{n}}{\partial t}(t, x)+\frac{1}{2} \sigma_{t}^{2} x^{2} \frac{\partial^{2} u^{n}}{\partial x^{2}}(t, x)\right. \\
\left.+r_{t} x \frac{\partial u^{n}}{\partial x}(t, x)-r_{t} u^{n}(t, x)\right)=0, \\
(t, x) \in\left(T_{n}, T\right) \times \mathbb{R}, \\
u^{n}(T, x)=\max (C x, F), \quad x \in \mathbb{R}, \\
\min \left(u^{i}(t, x)-C x, \frac{\partial u^{i}}{\partial t}(t, x)+\frac{1}{2} \sigma_{t}^{2} x^{2} \frac{\partial^{2} u^{i}}{\partial x^{2}}(t, x)\right. \\
\left.+r_{t} x \frac{\partial u^{i}}{\partial x}(t, x)-r_{t} u^{i}(t, x)\right)=0, \\
(t, x) \in\left(T_{i}, T_{i+1}\right) \times \mathbb{R}, \\
u^{i}\left(T_{i+1}, x\right)=\max \left(C x, u^{i+1}\left(T_{i+1},(1-\delta) x\right)\right), \quad x \in \mathbb{R},
\end{gathered}
$$

for $i=0,1, \ldots, n-1$ and $T_{0}=0$.

Theorem 10. Let Assumptions 5 and 9 hold. Then, the convertible bond price is given by $u^{i}\left(t, P_{t}\right)$, where $u^{i}(t, x)$ is determined by (34) and (35) successively, $i=0,1, \ldots, n . P_{t}$ is the stock price at time $t$.

\section{The Pricing Model with Higher Borrowing Rate}

In this section, let us investigate a more realistic case in the financial market, that is as follows.

Assumption 11. The borrowing rate $R_{t}$ is higher than the interest rate $r_{t}$, where $R_{t}$ is also deterministic and bounded.

Then analogous to the discussion in Section 3.1, suppose that the dividends is still paid continuously at the rate $d\left(t, P_{t}\right)$.
Then for any fixed $t \in[0, T]$ and $v \in \mathscr{T}_{t}$, there exists a unique hedging portfolio strategy $\left(\widetilde{X}_{s}\left(v, \widetilde{P}_{v}\right), \tilde{\pi}_{s}\left(v, \widetilde{P}_{v}\right)\right) \in \mathcal{S}_{\mathscr{F}}^{2} \times \mathscr{L}_{\mathscr{F}}^{2}$, denoted also by $\left(\widetilde{X}_{s}^{v}, \widetilde{\pi}_{s}^{v}\right)$ replicating $\widetilde{P}_{v}$; that is

$$
\begin{gathered}
-d \widetilde{X}_{s}^{v}=-\left[r_{s} \widetilde{X}_{s}^{v}+\left(\mu_{s}-r_{s}+d\left(s, P_{s}\right)\right) \tilde{\pi}_{s}^{v}\right. \\
\left.-\left(R_{s}-r_{s}\right)\left(\widetilde{X}_{s}^{v}-\widetilde{\pi}_{s}^{v}\right)^{-}\right] d s-\sigma_{s} \tilde{\pi}_{s}^{v} d W_{s}, \\
\widetilde{X}_{v}^{v}=\widetilde{P}_{v} .
\end{gathered}
$$

Thus, the price of convertible bond is given by the rightcontinuous adapted process $\left\{\widetilde{X}_{t}, 0 \leq t \leq T\right\}$ satisfying at each time $t$

$$
\widetilde{X}_{t}=\operatorname{ess} \sup _{v \in \mathscr{T}_{t}} \widetilde{X}_{t}\left(v, \widetilde{P}_{v}\right)
$$

Again by Proposition 3, it follows that the price process $\left\{\widetilde{X}_{t}, 0 \leq t \leq T\right\}$ corresponds to the solution of a reflected BSDE.

Theorem 12. Let Assumptions 5, 6, and 11 hold. Then, there exist $\left(\widetilde{\pi}_{t}\right) \in \mathscr{L}_{\mathscr{F}}^{2}$ and $\left(\widetilde{K}_{t}\right)$ an increasing adapted continuous process with $\widetilde{K}_{0}=0$ such that

$$
\begin{gathered}
\widetilde{X}_{t}=\xi+\int_{t}^{T}-\left[r_{s} \widetilde{X}_{s}^{v}+\left(\mu_{s}-r_{s}+d\left(s, P_{s}\right)\right) \widetilde{\pi}_{s}^{v}\right. \\
\left.-\left(R_{s}-r_{s}\right)\left(\widetilde{X}_{s}^{v}-\tilde{\pi}_{s}^{v}\right)^{-}\right] d s \\
+\widetilde{K}_{T}-\widetilde{K}_{t}-\int_{t}^{T} \sigma_{s} \tilde{\pi}_{s}^{v} d W_{s}, \quad 0 \leq t \leq T,
\end{gathered}
$$

where $\widetilde{X}_{t} \geq C P_{t}, 0 \leq t \leq T$, and $\int_{0}^{T}\left(\widetilde{X}_{t}-C P_{t}\right) d \widetilde{K}_{t}=0$.

Furthermore, the stopping time $\widetilde{D}_{t}=\inf \left\{t \leq s \leq T ; \widetilde{X}_{s}=\right.$ $\left.C P_{s}\right\}$ is the execution time of the convertible bond; that is

$$
\widetilde{X}_{t}=\operatorname{ess} \sup _{v \in \mathscr{T}_{t}} \widetilde{X}_{t}\left(v, \widetilde{P}_{v}\right)=\widetilde{X}_{t}\left(\widetilde{D}_{t}, \widetilde{P}_{\widetilde{D}_{t}}\right) .
$$

Combining Theorem 4, we easily obtain the following.

Theorem 13. Let Assumptions 5, 6, and 11 hold. Then, the following PDE

$$
\begin{gathered}
\min \left(\tilde{u}(t, x)-C x, \frac{\partial \tilde{u}}{\partial t}(t, x)+\frac{1}{2} \sigma_{t}^{2} x^{2} \frac{\partial^{2} \tilde{u}}{\partial x^{2}}(t, x)\right. \\
+\left(r_{t}-d(t, x)\right) x \frac{\partial \tilde{u}}{\partial x}(t, x)-r_{t} \tilde{u}(t, x) \\
\left.+\left(R_{t}-r_{t}\right)\left(\tilde{u}-x \frac{\partial \tilde{u}}{\partial x}\right)^{-}(t, x)\right)=0 \\
(t, x) \in(0, T) \times \mathbb{R} \\
\widetilde{u}(T, x)=\max (C x, F), \quad x \in \mathbb{R},
\end{gathered}
$$

admits a unique viscosity solution $\widetilde{u}(t, x)$. The convertible bond price process $\left\{\widetilde{X}_{t}, 0 \leq t \leq T\right\}$ can be given as

$$
\widetilde{X}_{t}=\tilde{u}\left(t, P_{t}\right), \quad \forall t \in[0, T],
$$

where $P_{t}$ is the price of underlying stock at time $t$. 
Remark 14. For the case that the dividends are paid discretely as in Assumption 9, one can easily derive the pricing formula for convertible bonds with higher borrowing rate by adopting the same methods stated previously. Thus, we just omit it here.

\section{Numerical Computations and Analysis}

From Theorems 8, 10, and 13, we can obtain the convertible bonds price in different situations by solving (24), (34), (35), and (40), respectively. However, there is no available analytical formula for the solutions of these PDEs. So we adopt the radial basis function (RBF) methods to solve PDEs and then present some examples to illustrate our theoretical results in above sections.

For simplicity, all functions $R_{t}, r_{t}, \mu_{t}, \sigma_{t}$, and $d(t, \cdot)$ are assumed to be constants in this section. We take PDE (24), for example, to introduce the RBF methods as follows. Let us first consider a PDE:

$$
\begin{aligned}
& \frac{\partial u}{\partial t}(t, x)+\frac{1}{2} \sigma^{2} x^{2} \frac{\partial^{2} u}{\partial x^{2}}(t, x) \\
& +(r-d) x \frac{\partial u}{\partial x}(t, x)-r u(t, x)=0 \\
& (t, x) \in(0, T) \times \mathbb{R} \\
& u(T, x)=\max (C x, F), \quad x \in \mathbb{R} .
\end{aligned}
$$

A simple transformation of $x=e^{y}$ changes (42) to

$$
\begin{gathered}
\frac{\partial u}{\partial t}(t, y)+\frac{1}{2} \sigma^{2} \frac{\partial^{2} u}{\partial y^{2}}(t, y) \\
+\left(r-d-\frac{1}{2} \sigma^{2}\right) \frac{\partial u}{\partial y}(t, y)-r u(t, y)=0, \\
(t, y) \in(0, T) \times \mathbb{R} \\
u(T, y)=\max \left(C e^{y}, F\right), \quad x \in \mathbb{R} .
\end{gathered}
$$

The idea of the proposed numerical scheme is to interpolate the unknown function $u$ by the following radial function $\phi$ :

$$
u(t, y) \simeq \sum_{j=1}^{N} \alpha_{j}(t) \phi\left(\left|y-y_{j}\right|\right)
$$

where $\alpha_{j}$ are unknown coefficients depending on time and $\phi_{j}(y)=\phi\left(\left|y-y_{j}\right|\right)$ are called RBFs because $\left|y-y_{j}\right|$ denotes the radial distance of each of the $N$ scattered data points $y_{j}$, $j=1,2, \ldots, N$. As we mentioned before, in this paper, we take Hardy's Multiquadric, as the radial basis function for the computation scheme. Thus

$$
\phi_{j}(y)=\sqrt{\left(y-y_{j}\right)^{2}+c^{2}}
$$

where $c$ is a positive constant called the shape parameter.
Collocating at the same $N$ points $y_{j}$ by substituting (44) into (43), we obtain the following system of linear equations, for $i=1,2, \ldots, N$,

$$
\begin{aligned}
& \frac{\partial u}{\partial t}\left(t, y_{i}\right)+\frac{1}{2} \sigma^{2} \frac{\partial^{2} u}{\partial y^{2}}\left(t, y_{i}\right) \\
& +\left(r-d-\frac{1}{2} \sigma^{2}\right) \frac{\partial u}{\partial y}\left(t, y_{i}\right)-r u\left(t, y_{i}\right)=0
\end{aligned}
$$

Since $\phi_{j}(y)$ does not depend on time, the time derivative of $u$ is simply given in terms of the time derivatives of the coefficients:

$$
\frac{\partial u}{\partial t}\left(t, y_{i}\right)=\sum_{j=1}^{N} \frac{d \alpha_{j}(t)}{d t} \phi\left(\left|y_{i}-y_{j}\right|\right) .
$$

The first and second partial derivatives of $u$ with respect to $y$ are given, respectively, as follows:

$$
\begin{aligned}
\frac{\partial u}{\partial y}\left(t, y_{i}\right) & =\sum_{j=1}^{N} \alpha_{j}(t) \frac{\partial \phi}{\partial y}\left(\left|y_{i}-y_{j}\right|\right), \\
\frac{\partial^{2} u}{\partial y^{2}}\left(t, y_{i}\right) & =\sum_{j=1}^{N} \alpha_{j}(t) \frac{\partial^{2} \phi}{\partial y^{2}}\left(\left|y_{i}-y_{j}\right|\right),
\end{aligned}
$$

in which

$$
\frac{\partial \phi}{\partial y}\left(\left|y_{i}-y_{j}\right|\right)=\frac{y_{i}-y_{j}}{\sqrt{\left(y_{i}-y_{j}\right)^{2}+c^{2}}}
$$

$$
\begin{aligned}
\frac{\partial^{2} \phi}{\partial y^{2}}\left(\left|y_{i}-y_{j}\right|\right)= & \frac{1}{\sqrt{\left(y_{i}-y_{j}\right)^{2}+c^{2}}} \\
& -\frac{\left(y_{i}-y_{j}\right)^{2}}{\left(\sqrt{\left(y_{i}-y_{j}\right)^{2}+c^{2}}\right)^{3 / 2}} .
\end{aligned}
$$

Thus, in matrix form, (46) can be expressed as

$$
L \dot{\alpha}+\frac{1}{2} \sigma^{2} L_{y y} \alpha+\left(r-d-\frac{1}{2} \sigma^{2}\right) L_{y} \alpha-r L \alpha=0,
$$

where $\alpha$ denotes the vector containing the unknown coefficients $\alpha_{j}(t), \dot{\alpha}$ the time derivatives, and $L, L_{y}$, and $L_{y y}$ are $N \times N$ matrices with entries $\phi\left(\left|y_{i}-y_{j}\right|\right),(\partial \phi / \partial y)\left(\mid y_{i}-\right.$ $\left.y_{j} \mid\right)$, and $\left(\partial^{2} \phi / \partial y^{2}\right)\left(\left|y_{i}-y_{j}\right|\right)$ given by (45), (49), and (50), respectively. It has been proven by Powell [20] that the matrix $L$ is invertible for distinct collocation points $y_{j}$, and hence $L^{-1}$ exists. Thus, (51) can be rewritten as

$$
\dot{\alpha}=-L^{-1}\left[\frac{1}{2} \sigma^{2} L_{y y} \alpha+\left(r-d-\frac{1}{2} \sigma^{2}\right) L_{y} \alpha-r L \alpha\right] \equiv G \alpha,
$$

where $G$ is the $N \times N$ matrix

$$
G=r I-\frac{1}{2} \sigma^{2} L^{-1} L_{y y}-\left(r-d-\frac{1}{2} \sigma^{2}\right) L^{-1} L_{y},
$$

with $I$ being the identity matrix of size $N$. 
For fixed collocation points $y_{j}, j=1,2, \ldots, N,(52)$ is a linear system of first-order homogeneous ordinary differential equations with constant coefficients, with the terminal condition

$$
\alpha(T)=L^{-1}\left(u\left(T, y_{1}\right), u\left(T, y_{2}\right), \ldots, u\left(T, y_{N}\right)\right)^{T} \equiv L^{-1} U,
$$

where $U=\left(u\left(T, y_{1}\right), u\left(T, y_{2}\right), \ldots, u\left(T, y_{N}\right)\right)^{T}$, whose value is given by (43). Then, we can use explicit second-order backward time integration scheme to obtain the unknown coefficients $\alpha$ at each time step $T-\Delta t$. For notational convenience, let $U^{n}=L \alpha^{n}$ denote the approximation $U(T-$ $\left.n \Delta t, y_{i}\right)$ at each time step $T-n \Delta t$. We have

$$
\begin{gathered}
F_{1}=-\Delta t G \alpha^{n-1}, \\
F_{2}=-\Delta t G\left(\alpha^{n-1}+0.5 F_{1}\right), \\
\alpha^{n}=\alpha^{n-1}+0.5\left(F_{1}+F_{2}\right) .
\end{gathered}
$$

Thus, the solution of (42) can be approximated by (44).

Now let us turn to (24). From the different forms of (24) and (42), it is easily seen that the evaluation of (24) can be performed by modifying the above procedure as follows.

Step 1: compute $U^{n}=L \alpha^{n}$.

Step 2: for $i=1,2, \ldots, N$, update the $i$ th element $U^{n}(i)=$ $\max \left(C e^{y_{i}}, U^{n}(i)\right)$.

Step 3: The updated $\alpha^{n}$ is simply $L^{-1} U^{n}$.

Then, the solution of (24) can also be approximated by (44).

Remark 15. For PDEs (34), (35), and (40), one can easily proceed the evaluation similarly as above. Here, we do not give the detailed derivation any more.

Remark 16. The accuracy of MQ is greatly affected by the choice of the shape parameter $c$, and an optimal recipe for the value of $c$ is still under intensive research. We adopt the suggestion from Hardy [16] by choosing $c$ to be $4 d_{\text {min }}$, where $d_{\text {min }}$ is the minimum distance between any two collocation points $y_{j}$.

Now, let us present some examples of pricing the convertible bonds by above numerical calculation and discuss the influence of dividends as well as the higher borrowing rate.

Example 17. Suppose for four convertible bonds, $C=4.36$, $F=1000$ yuan, $r=0.1121, \sigma=0.30$, and $T=15$ years are the same, while their underlying stocks pay dividends at different rates $d_{1}=0, d_{2}=0.005, d_{3}=0.010$, and $d_{4}=0.016$. Then according to Theorem 8 , computing (24) by RBF method, we obtain their prices corresponding to the initial stock prices varying from 52.00 to 54.00 yuan as shown in Table 1 .

From Table 1, we can see that the continuous dividends paying lower the price of convertible bond. The larger the dividends rate is, the lower the convertible bond price is. From the point of view of economics, the actual value
TABLE 1: The price of convertible bond with different dividends rates.

\begin{tabular}{lcccc}
\hline \multirow{2}{*}{ Stock price } & \multicolumn{4}{c}{ Dividend rate } \\
& $d_{1}=0.000$ & $d_{2}=0.005$ & $d_{3}=0.010$ & $d_{4}=0.016$ \\
\hline 52.00 & 297.0862 & 288.5835 & 281.1576 & 273.7534 \\
52.25 & 297.9239 & 289.3795 & 281.9206 & 274.4878 \\
52.50 & 298.7629 & 290.1769 & 282.6853 & 275.2241 \\
52.75 & 299.6033 & 290.9758 & 283.4515 & 275.9623 \\
53.00 & 300.4450 & 291.7761 & 284.2194 & 276.7023 \\
53.25 & 301.2880 & 292.5778 & 284.9889 & 277.4442 \\
53.50 & 302.1323 & 293.3810 & 285.7600 & 278.1880 \\
53.75 & 302.9779 & 294.1856 & 286.5326 & 278.9335 \\
54.00 & 303.8248 & 294.9916 & 287.3069 & 279.6810 \\
\hline
\end{tabular}

TABLE 2: The price of convertible bond with different dividends proportions.

\begin{tabular}{lcccc}
\hline \multirow{2}{*}{ Stock price } & \multicolumn{4}{c}{ Dividend proportion } \\
& $\delta_{1}=0.000$ & $\delta_{2}=0.005$ & $\delta_{3}=0.010$ & $\delta_{4}=0.016$ \\
\hline 52.00 & 297.0862 & 289.1203 & 282.2663 & 275.4526 \\
52.25 & 297.9239 & 289.9201 & 283.0373 & 276.2003 \\
52.50 & 298.7629 & 290.7213 & 283.8099 & 276.9499 \\
52.75 & 299.6033 & 291.5239 & 284.5842 & 277.7014 \\
53.00 & 300.4450 & 292.3281 & 285.3601 & 278.4548 \\
53.25 & 301.2880 & 293.1336 & 286.1376 & 279.2100 \\
53.50 & 302.1323 & 293.9406 & 286.9167 & 279.9672 \\
53.75 & 302.9779 & 294.7490 & 287.6975 & 280.7262 \\
54.00 & 303.8248 & 295.5588 & 288.4798 & 281.4871 \\
\hline
\end{tabular}

of stocks will decrease for the dividends paying. Then by the comparison theorem of reflected BSDEs, the price of convertible bond will indeed lower correspondingly.

Example 18. Suppose for four convertible bonds, their underlying stocks pay dividends in different proportions $\delta_{1}=0$, $\delta_{2}=0.005, \delta_{3}=0.010$, and $\delta_{4}=0.016$ of the prices at the end of every year after issuing, while other features like $C, F, r, \sigma$, and $T$ are the same as those in Example 17. Then according to Theorem 10, computing (34) and (35) successively by RBF method, we obtain their prices corresponding to the initial stock prices varying from 52.00 to 54.00 yuan as shown in Table 2 .

This example shows that how the discrete dividends paying affects the prices of convertible bonds. The stock price will fall down explicitly after every time the dividends being paid. As the explanation of Example 17, it is reasonable for the convertible bond price to decline accordingly.

At last, let us see the influence of higher borrowing rate on the convertible bond price.

Example 19. Suppose all features about the convertible bonds keep the same with Example 17, while the borrowing rate $R=0.150$, which is higher than the interest rate $r$ in the financial market. Then according to Theorem 13, computing (40) by RBF method, we obtain their prices corresponding to the initial stock prices varying from 52.00 to 54.00 yuan as shown in Table 3. 
TABLE 3: The price of convertible bond with higher borrowing rate.

\begin{tabular}{lcccc}
\hline \multirow{2}{*}{ Stock price } & \multicolumn{4}{c}{ Dividend rate } \\
& $d_{1}=0.000$ & $d_{2}=0.005$ & $d_{3}=0.010$ & $d_{4}=0.016$ \\
\hline 52.00 & 297.0862 & 288.5835 & 281.1576 & 273.7534 \\
52.25 & 297.9239 & 289.3795 & 281.9206 & 274.4878 \\
52.50 & 298.7629 & 290.1769 & 282.6853 & 275.2241 \\
52.75 & 299.6033 & 290.9758 & 283.4515 & 275.9623 \\
53.00 & 300.4450 & 291.7761 & 284.2194 & 276.7023 \\
53.25 & 301.2880 & 292.5778 & 284.9889 & 277.4442 \\
53.50 & 302.1323 & 293.3810 & 285.7600 & 278.1880 \\
53.75 & 302.9779 & 294.1856 & 286.5326 & 278.9335 \\
54.00 & 303.8248 & 294.9916 & 287.3069 & 279.6810 \\
\hline
\end{tabular}

Comparing Table 3 with Table 1, it is an interesting fact that the higher borrowing rate has no influence on the prices of convertible bonds. When the borrowing rate $R$ is up to 0.200 , one can obtain the same result. It means that, in order to hedge the contingent claim of convertible bond, one need not to borrow money from the bank; that is, the money invested into the stock market is always less than total wealth of the investor. Wang and $\mathrm{Wu}$ [21] have proved that this fact is always true when there are no dividends by means of Malliavin derivatives and comparison theorem for BSDEs. However, it needs more investigation for whether it still holds with dividends being paid.

\section{Conclusion and Extension}

It is the first attempt to formulate the pricing model for convertible bonds with dividends paying of their underlying stocks via reflected BSDEs, to the authors' knowledge. Compared with existing literatures devoting to this problem, there are three distinguishing features of our paper: (1) we consider the situation that underlying stocks pay dividends both continuously and discretely and the borrowing rate is higher than interest rate; (2) we establish the pricing formula for convertible bonds by the solution of obstacle problems for some PDEs; (3) we present a numerical evaluation method, the RBFs, to evaluate the convertible bond prices, illustrating the applications of our theoretical study.

In our numerical examples, we see that the higher borrowing rate has no effect on the price of convertible bond. However, it begs systematic study to prove whether this fact is always true no matter the dividends is paid continuously or discretely, which seems related to the field of Malliavin derivatives. We hope to develop some theories for this topic. Besides, during the numerical procedure, all coefficients are confined to constants, and the accuracy and stability of RBF methods need more investigation and improvement. We are also looking forward to a modified method, which can deal with time-dependent or even random coefficients.

\section{Acknowledgments}

The authors would like to thank Professor Zhen $\mathrm{Wu}$ for his enthusiastic discussions and enlightened suggestions related to this work. Also the authors are grateful to anonymous referees and editors for their valuable comments, which led to an improved version of this paper. This work is supported by the Natural Science Foundation of China (10921101 and 61174092).

\section{References}

[1] O. H. Poensgen, "The valuation of convertible bonds, part 1," Industrial Management Review, vol. 7, pp. 77-92, 1965.

[2] O. H. Poensgen, "The valuation of convertible bonds, part 2," Industrial Management Review, vol. 7, pp. 83-98, 1966.

[3] W. J. Baumol, B. G. Malkiel, and R. E. Quandt, "The valuation of convertible securities," Quarterly Journal of Economics, vol. 40, pp. 48-59, 1966.

[4] R. Weil, J. E. Segall, and D. Green, "Premiums on convertible bonds," Journal of Finance, vol. 23, pp. 445-463, 1968.

[5] F. Black and M. Scholes, "The pricing of options and corporate liabilities," Journal of Political Economy, vol. 81, pp. 637-654, 1973.

[6] J. E. Ingersoll, "A contingent-claims valuation of convertible securities," Journal of Financial Economics, vol. 4, pp. 289-382, 1977.

[7] M. J. Brennan and E. S. Schwartz, "Convertible bonds: valuation and optimal strategies for call and conversion," Journal of Finance, vol. 32, pp. 1699-1715, 1977.

[8] M. J. Brennan and E. S. Schwartz, "Analyzing convertible bonds," Journal of Financal and Quantitative Analysis, vol. 15, pp. 907-929, 1980.

[9] J. J. McConnell and E. S. Schwartz, "LYON taming," Journal of Finance, vol. 41, pp. 561-576, 1986.

[10] T. S. Y. Ho and D. M. Pfeffer, "Convertible bonds: model, value attribution, and analytics," Financial Analysts Journal, vol. 52, pp. 35-44, 1996.

[11] K. Tsiveriotis and C. Fernandes, "Valuing convertible bonds with credit risk," Journal of Fixed Income, vol. 8, pp. 95-102, 1998.

[12] E. Pardoux and S. G. Peng, "Adapted solution of a backward stochastic differential equation," Systems and Control Letters, vol. 14, no. 1, pp. 55-61, 1990.

[13] D. Duffie and L. G. Epstein, "Stochastic differential utility," Econometrica, vol. 60, no. 2, pp. 353-394, 1992.

[14] N. El Karoui, S. G. Peng, and M. C. Quenez, "Backward stochastic differential equations in finance," Mathematical Finance, vol. 7, no. 1, pp. 1-71, 1997.

[15] N. El Karoui, C. Kapoudjian, E. Pardoux, S. G. Peng, and M. C. Quenez, "Reflected solutions of backward SDE's, and related obstacle problems for PDE's," The Annals of Probability, vol. 25, no. 2, pp. 702-737, 1997.

[16] R. L. Hardy, "Multiquadric equations of topography and other irregular surfaces," Journal of Geophysical Research, vol. 76, pp. 1905-1915, 1971.

[17] R. Franke, "Scattered data interpolation: tests of some methods," Mathematics of Computation, vol. 38, no. 157, pp. 181-200, 1982.

[18] Y. C. Hon and X. Z. Mao, "A radial basis function method for solving options pricing models," Financial Engineering, vol. 8, pp. 31-50, 1999.

[19] N. El Karoui, E. Pardoux, and M. C. Quenez, "Reected backward SDEs and American options," Numerical Methods in Finance, vol. 6, pp. 215-231, 1997. 
[20] M. J. D. Powell, "The theory of radial basis function approximation," in Advances in Numerical Analysis, vol. 3, pp. 105-210, Oxford University Press, New York, NY, USA, 1992.

[21] H. Y. Wang and Z. Wu, "Backward stochastic differential equations and application in pricing the convertible bond," Journal of Mathematical Analysis and Applications. In press. 


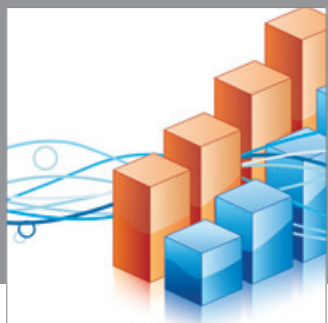

Advances in

Operations Research

mansans

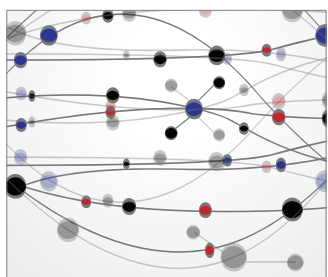

The Scientific World Journal
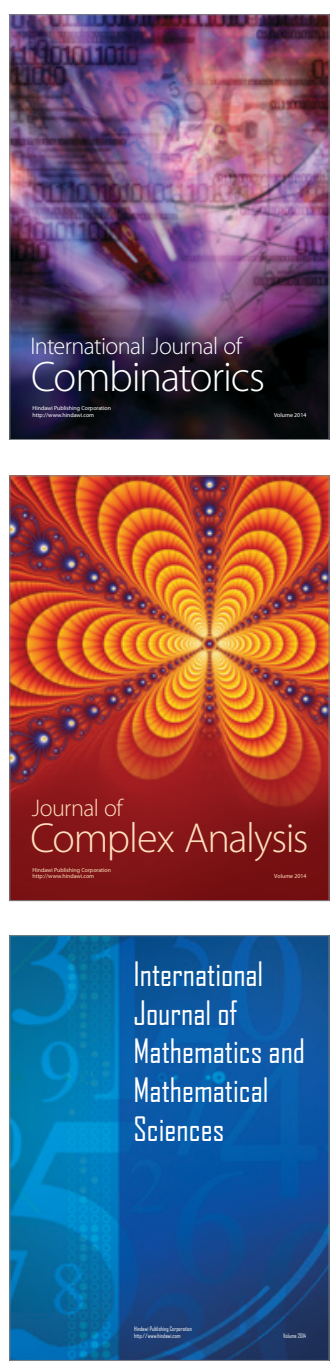
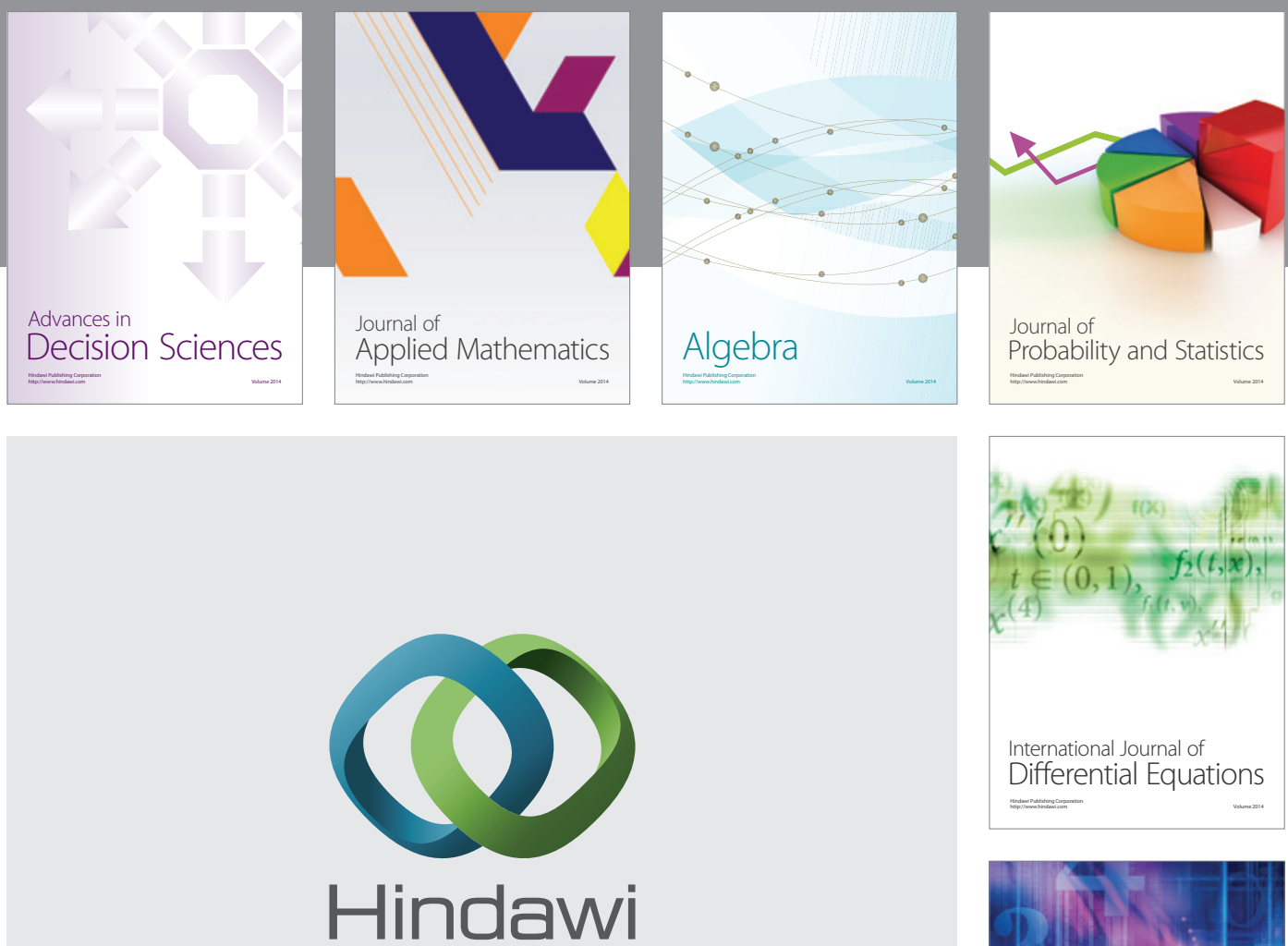

Submit your manuscripts at http://www.hindawi.com
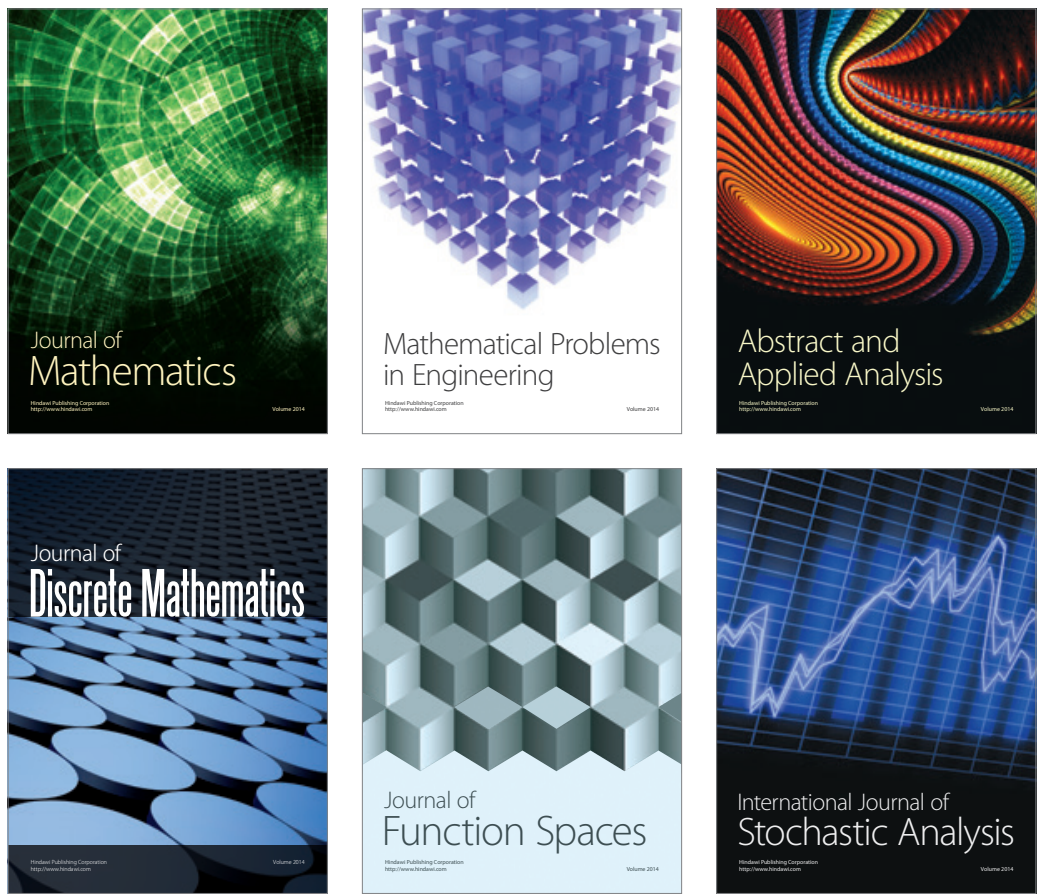

Journal of

Function Spaces

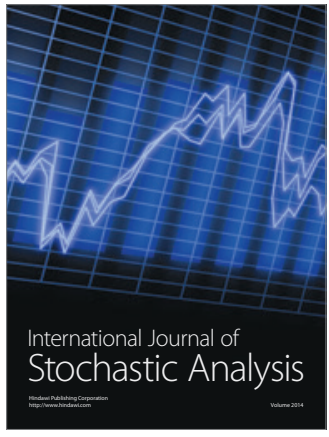

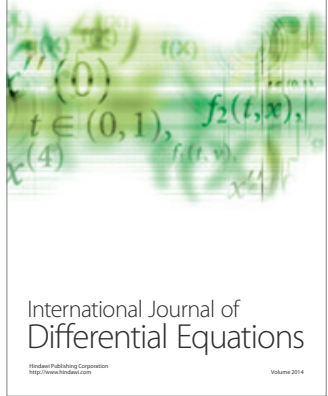
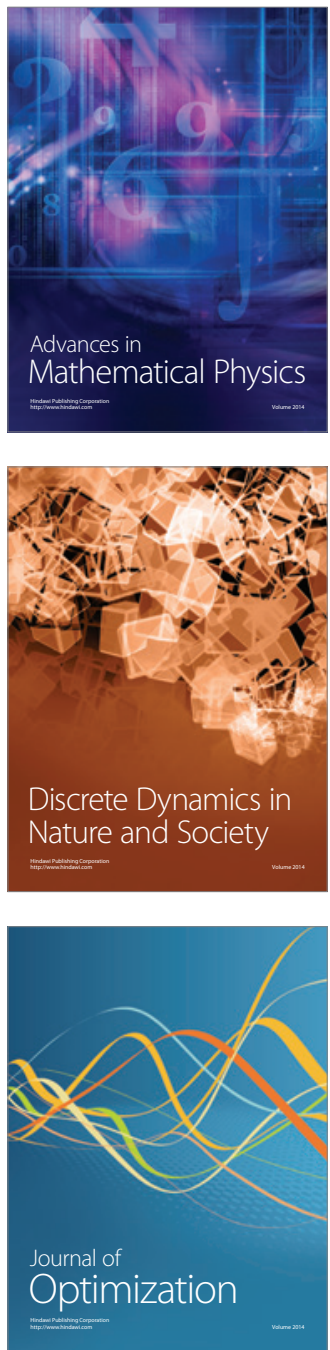